\title{
PERIODIC SOLUTIONS FOR A FRACTIONAL ASYMPTOTICALLY LINEAR PROBLEM
}

\author{
VINCENZO AMBROSIO AND GIOVANNI MOLICA BISCI
}

\begin{abstract}
We study the existence and multiplicity of periodic weak solutions for a non-local equation involving an odd subcritical nonlinearity which is asymptotically linear at infinity. We investigate such problem by applying the the pseudo-index theory developed by Bartolo, Benci and Fortunato [9] after transforming the problem to a degenerate elliptic problem in a half-cylinder with a Neumann boundary condition, via a Caffarelli-Silvestre type extension in periodic setting. The periodic nonlocal case, considered here, presents, respect to the cases studied in literature, some new additional difficulties and a careful analysis of the fractional spaces involved is necessary.
\end{abstract}

\section{INTRODUCTION}

We consider here the non-local counterpart in periodic setting of the semilinear problem

$$
\begin{cases}-\Delta u=g(x, u) & \text { in } \Omega \\ u=0 & \text { on } \partial \Omega,\end{cases}
$$

where $\Omega \subset \mathbb{R}^{N}$ is a smooth bounded domain and $g: \Omega \times \mathbb{R} \rightarrow \mathbb{R}$ is a given function asymptotically linear and possibly odd.

We notice that problem (1.1) has been widely studied in the past years by many authors by using topological and variational methods (see, among others, $[1,9,32]$ and references therein).

In recent years, great attention has been devoted to the study of elliptic problems involving non-local operators which arise in a quite natural way in several areas of research; for more details and applications we refer to [20] and the recent book [26].

However, although in literature there are many papers dealing with non-local fractional Laplacian equations with superlinear and sublinear growth $[2,3,4,6,7,8,37,38]$, only few papers consider asymptotically linear problems in fractional setting, see, for instance, [12, 13, 15, 22, 27].

Key words and phrases. Fractional Laplacian; Variational methods; Periodic solutions; Asymptotically linear problem; Nonresonant problems; Pseudo-genus. 
The aim of the present paper is to give a further result in this direction, considering a non-local problem with periodic boundary conditions.

More precisely, we are interested in the existence and multiplicity of the following problem

$$
\begin{cases}\left(-\Delta+m^{2}\right)^{s} u=\lambda_{\infty} u+f(x, u) & \text { in }(0, T)^{N} \\ u\left(x+T e_{i}\right)=u(x) & \forall x \in \mathbb{R}^{N}, i \in \mathbb{Z}[1, N]\end{cases}
$$

where $s \in(0,1), N \geqslant 2, m>0, \lambda_{\infty} \in \mathbb{R},\left\{e_{i}\right\}_{i=1}^{N}$ is the canonical basis in $\mathbb{R}^{N}, f: \mathbb{R}^{N} \times \mathbb{R} \rightarrow \mathbb{R}$ is a Caratheodory function satisfying suitable assumptions, and $\mathbb{Z}[1, N]:=\{1, \ldots, N\}$.

Moreover, the nonlocal operator $\left(-\Delta+m^{2}\right)^{s}$ appearing in (1.2) is defined by setting

$$
\left(-\Delta+m^{2}\right)^{s} u(x):=\sum_{k \in \mathbb{Z}^{N}} \beta_{k}\left(\omega^{2}|k|^{2}+m^{2}\right)^{s} \frac{e^{\imath \omega k \cdot x}}{\sqrt{T^{N}}},
$$

for any $u=\sum_{k \in \mathbb{Z}^{N}} \beta_{k} \frac{e^{\imath \omega k \cdot x}}{\sqrt{T^{N}}} \in \mathcal{C}_{T}^{\infty}\left(\mathbb{R}^{N}\right)$, where

$$
\omega:=\frac{2 \pi}{T} \text { and } \beta_{k}:=\frac{1}{\sqrt{T^{N}}} \int_{(0, T)^{N}} u(x) e^{-\imath \omega k \cdot x} d x \quad\left(k \in \mathbb{Z}^{N}\right)
$$

are the Fourier coefficients of the smooth and $T$-periodic function $u$.

This operator can be extended by density for every function that lies in the Hilbert space

$\mathbb{H}_{T}^{s}:=\left\{u=\sum_{k \in \mathbb{Z}^{N}} \beta_{k} \frac{e^{\imath \omega k \cdot x}}{\sqrt{T^{N}}} \in L^{2}(0, T)^{N}: \sum_{k \in \mathbb{Z}^{N}}\left(\omega^{2}|k|^{2}+m^{2}\right)^{s}\left|\beta_{k}\right|^{2}<+\infty\right\}$

endowed by the norm

$$
|u|_{\mathbb{H}_{T}^{s}}:=\left(\sum_{k \in \mathbb{Z}^{N}}\left(\omega^{2}|k|^{2}+m^{2}\right)^{s}\left|\beta_{k}\right|^{2}\right)^{1 / 2}
$$

We also recall the embedding properties of $\mathbb{H}_{T}^{s}$ into the usual Lebesgue spaces; see Theorems 3 and 4 in Subsection 2.1. The embedding $j: \mathbb{H}_{T}^{s} \hookrightarrow L^{\nu}(0, T)^{N}$ is continuous for any $\nu \in\left[1,2_{s}^{\sharp}\right.$, while it is compact whenever $\nu \in\left[1,2_{s}^{\sharp}\right)$, where $2_{s}^{\sharp}:=2 N /(N-2 s)$ denotes the fractional critical Sobolev exponent.

From a physical point of view, the meaning of the fractional operator $\left(-\Delta+m^{2}\right)^{s}$ is manifest in the case $s=\frac{1}{2}$; in such case, $\left(-\Delta+m^{2}\right)^{1 / 2}-m$ is the so-called free Hamiltonian which plays a fundamental role in relativistic quantum mechanic; see [25]. 
Furthermore, $\left(-\Delta+m^{2}\right)^{s}-m^{2 s}$ is also related to the Lévy Processes theory; the operator in question is an infinitesimal generator of the relativistic $2 s$-stable process $\left\{X_{t}^{m}\right\}_{t \geqslant 0}$ with characteristic function given by

$$
\mathbb{E}\left(e^{i \xi \cdot X_{t}^{m}}\right):=e^{-t\left[\left(m^{2}+|\xi|^{2}\right)^{s}-m^{2 s}\right]} \quad\left(\xi \in \mathbb{R}^{N}\right) ;
$$

we refer to [19] and [34].

From now on we will suppose that $f: \mathbb{R}^{N+1} \rightarrow \mathbb{R}$ is a Caratheodory function satisfying the following assumptions:

$\left(f_{1}\right) f(x, t)$ is $T$-periodic in $x \in \mathbb{R}^{N}$, that is $f\left(x+T e_{i}, t\right)=f(x, t)$ for any $x \in \mathbb{R}^{N}(T \in \mathbb{R})$, for every $i \in \mathbb{Z}[1, N]$ and

$$
\sup _{|t| \leqslant a}|f(\cdot, t)| \in L^{\infty}(0, T)^{N} \text { for any } a>0 ;
$$

$\left(f_{2}\right)$ there exist

$$
\lim _{|t| \rightarrow \infty} \frac{f(x, t)}{t}=0
$$

and

$$
\lim _{t \rightarrow 0} \frac{f(x, t)}{t}=\lambda_{0} \in \mathbb{R}
$$

uniformly with respect to a.e. $x \in \mathbb{R}^{N}$.

Let us denote respectively by $\sigma\left(\left(-\Delta+m^{2}\right)^{s}\right)$ and by

$$
0<\lambda_{1}<\lambda_{2} \leqslant \ldots \leqslant \lambda_{k} \leqslant \ldots
$$

the spectrum and the non-decreasing, diverging sequence of the following problem

$$
\begin{cases}\left(-\Delta+m^{2}\right)^{s} u=\lambda u & \text { in }(0, T)^{N} \\ u\left(x+T e_{i}\right)=u(x) & \forall x \in \mathbb{R}^{N}, i \in \mathbb{Z}[1, N]\end{cases}
$$

repeated according to their multiplicity; by using the classical spectral theory $[33,41]$, in Subsection 2.2 we recall some features, which are very closed to the well known ones of $-\Delta$, about the spectrum of the operator $\left(-\Delta+m^{2}\right)^{s}$.

With the above notation, our first result can be stated as follows

Theorem 1. Let $m>0, s \in(0,1)$ and $N \geqslant 2$. Suppose that $f$ : $\mathbb{R}^{N+1} \rightarrow \mathbb{R}$ is a continuous function satisfying $\left(f_{1}\right)$ and $\left(f_{2}\right)$. Then, problem (1.2) has at least a weak solution in $\mathbb{H}_{T}^{s}$, provided that $\lambda_{\infty} \notin$ $\sigma\left(\left(-\Delta+m^{2}\right)^{s}\right)$.

The main difficulty in our approach is essentially based on the fact that problem (1.2) is of non-nonlocal type. In addition, it doesn't seem to satisfy boundary conditions which would allow a direct application of variational methods without a certain extension procedure (see Section 
2). Following the work of Caffarelli and Silvestre [18], the fractional Laplacian operator in the whole space $\mathbb{R}^{n}$ can be defined as a Dirichlet to a Neumann map:

$$
(-\Delta)^{s} u(x):=-\kappa_{s} \lim _{y \rightarrow 0^{+}} y^{1-2 s} \frac{\partial w}{\partial y}(x, y),
$$

where $\kappa_{s}$ is a suitable constant and $w$ is the $s$-harmonic extension of a smooth function $u$. In other words, $w$ is the function defined on the upper half-space $\mathbb{R}_{+}^{n+1}:=\mathbb{R}^{n} \times(0,+\infty)$ which is solution to the local elliptic problem

$$
\left\{\begin{array}{cl}
-\operatorname{div}\left(y^{1-2 s} \nabla w\right)=0 & \text { in } \mathbb{R}_{+}^{n+1} \\
w(x, 0)=u(x) & \text { in } \mathbb{R}^{n}
\end{array}\right.
$$

In order to define the fractional Laplacian operator in bounded domains, the above procedure has been adapted in $[16,17]$. To overcome the nonlocalicity of the operator $\left(-\Delta+m^{2}\right)^{s}$ in $(1.2)$, we use the type extension in periodic setting developed in $[2,3]$.

More precisely, for any $u \in \mathbb{H}_{T}^{s}$, there exists a unique $v \in \mathbb{X}_{T}^{s}$ which solves

$$
\begin{cases}-\operatorname{div}\left(y^{1-2 s} \nabla v\right)+m^{2} y^{1-2 s} v=0 & \text { in } \mathcal{S}_{T}:=(0, T)^{N} \times(0, \infty) \\ v_{\mid\left\{x_{i}=0\right\}}=v_{\mid\left\{x_{i}=T\right\}} & \text { on } \partial_{L} \mathcal{S}_{T}:=\partial(0, T)^{N} \times[0, \infty), \\ v(x, 0)=u(x) & \text { on } \partial^{0} \mathcal{S}_{T}:=(0, T)^{N} \times\{0\}\end{cases}
$$

where $\mathbb{X}_{T}^{s}$ is the closure of the set of smooth and $T$-periodic (in $x$ ) functions in $\mathbb{R}_{+}^{N+1}$ with respect to the norm

$$
\|v\|_{\mathbb{X}_{T}^{s}}:=\left(\iint_{\mathcal{S}_{T}} y^{1-2 s}\left(|\nabla v|^{2}+m^{2 s} v^{2}\right) d x d y\right)^{1 / 2} .
$$

Then, the operator $\left(-\Delta+m^{2}\right)^{s}$ is obtained as

$$
-\lim _{y \rightarrow 0^{+}} y^{1-2 s} \frac{\partial v}{\partial y}(x, y)=\kappa_{s}\left(-\Delta+m^{2}\right)^{s} u(x)
$$

in weak sense and $\kappa_{s}:=2^{1-2 s} \frac{\Gamma(1-s)}{\Gamma(s)}$.

Therefore, we exploit this fact, and instead of (1.2), we investigate the following problem

$$
\begin{cases}-\operatorname{div}\left(y^{1-2 s} \nabla v\right)+m^{2} y^{1-2 s} v=0 & \text { in } \mathcal{S}_{T} \\ v_{\mid\left\{x_{i}=0\right\}}=v_{\mid\left\{x_{i}=T\right\}} & \text { on } \partial_{L} \mathcal{S}_{T} \\ \partial_{\nu}^{1-2 s} v=\kappa_{s}\left[\lambda_{\infty} v+f(x, v)\right] & \text { on } \partial^{0} \mathcal{S}_{T}\end{cases}
$$

where

$$
\partial_{\nu}^{1-2 s} v(x):=-\lim _{y \rightarrow 0^{+}} y^{1-2 s} \frac{\partial v}{\partial y}(x, y)
$$


is the conormal exterior derivative of $v$.

Since (1.5) has a variational structure, its solutions can be found as critical points of the energy functional $\mathcal{J}$ given by

$$
\mathcal{J}(v):=\frac{1}{2}\left(\|v\|_{\mathbb{X}_{T}^{s}}^{2}-\lambda_{\infty} \kappa_{s}|\operatorname{Tr}(v)|_{L^{2}(0, T)^{N}}^{2}\right)-\kappa_{s} \int_{\partial^{0} \mathcal{S}_{T}} F(x, \operatorname{Tr}(v)) d x
$$

defined on the space $\mathbb{X}_{T}^{s}$.

A natural question is whether or not these topological and variational methods may be adapted to equation (1.2) and to its generalization in order to extend the classical results known for (1.1) to a non-local periodic context.

In the first part of the paper we prove that the geometry of the classical Saddle Point Theorem due to Rabinowitz [32] is respected by the non-local framework: for this we use a functional analytical setting that is inspired by (but not equivalent to) the fractional Sobolev spaces, in order to correctly encode the periodic boundary datum in the variational formulation. Of course, also the compactness property required by this abstract theorem is satisfied in the non-local setting, again thanks to the choice of the functional setting we work in.

In addition, when the nonlinear-term is symmetric, we obtain the existence of multiple periodic solutions to (1.2). More precisely, we adapt the pseudo-index theory due to Bartolo, Benci and Fortunato [9], to prove a multiplicity result for critical points of the even energy functional $\mathcal{J}$; see also $[10,11,12,13]$ for related topics.

More precisely, we are able to prove the following result

Theorem 2. Let $m>0, s \in(0,1)$ and $N \geqslant 2$. Suppose that $f$ : $\mathbb{R}^{N+1} \rightarrow \mathbb{R}$ is a continuous function satisfying conditions $\left(f_{1}\right)$ and $\left(f_{2}\right)$. Further, let us assume that $f(x, \cdot)$ is odd for a.e. $x \in \mathbb{R}^{N}$ and require that

$\left(C_{\lambda_{\infty}}\right)$ there exist $h, k \in \mathbb{N}$, with $k \geqslant h$, such that

$$
\lambda_{0}+\lambda_{\infty}<\lambda_{h} \leqslant \lambda_{k}<\lambda_{\infty}
$$

Then, problem (1.2) has at least $k-h+1$ distinct pairs of non-trivial weak solutions in $\mathbb{H}_{T}^{s}$, provided that $\lambda_{\infty} \notin \sigma\left(\left(-\Delta+m^{2}\right)^{s}\right)$.

We emphasize that further difficulties arise in the so-called "resonant case", that is $\lambda_{\infty} \in \sigma\left(\left(-\Delta+m^{2}\right)^{s}\right)$ : indeed, the resonance affects both the compactness property and the geometry of the Euler-Lagrange functional arising in a suitable variational approach (cf., e.g., [9] and references therein for the classical elliptic case). We will consider this interesting case via some further investigations. Finally, we also point 
out that our results should be viewed as a periodic nonlocal version of Proposition 1.1 and Theorem 1.2 of [13].

For the sake of completeness we point out that fractional nonlocal equations have by now been widely investigated from many points of view. Besides [7, 8, 17, 30, 31, 35, 36] we cite [23, 24, 28, 29] and the very recent paper [40] and references therein for some nice interpretations of nonlocal physical phenomena.

As far as we know the results presented here are new for periodic fractional problems. The body of the paper is as follows: in Section 2 we collect some preliminaries related to the functional setting and the abstract critical point theory that we use to study (1.5): in Section 3 we prove the existence of a weak solution to (1.2), while in the last Section 4 we give the proof of Theorem 2 .

\section{Preliminaries}

2.1. Functional setting. This section is devoted to the notations used along the present paper. In order to give the weak formulation of problem (1.2), we need to work in a special functional space. Indeed, one of the difficulties in treating problem (1.2) is related to encoding the periodic boundary condition in the variational formulation. With this respect the standard fractional Sobolev spaces are not sufficient in order to study the problem. We overcome this difficulty by working in a new functional space, whose definition is recalled here. From now on, we assume $s \in(0,1)$ and $N \geqslant 2$.

Let

$$
\mathbb{R}_{+}^{N+1}=\left\{(x, y) \in \mathbb{R}^{N+1}: x \in \mathbb{R}^{N}, y>0\right\}
$$

be the upper half-space in $\mathbb{R}^{N+1}$.

Let $\mathcal{S}_{T}:=(0, T)^{N} \times(0, \infty)$ be the half-cylinder in $\mathbb{R}_{+}^{N+1}$ with basis $\partial^{0} \mathcal{S}_{T}:=(0, T)^{N} \times\{0\}$ and we denote by $\partial_{L} \mathcal{S}_{T}:=\partial(0, T)^{N} \times[0,+\infty)$ the lateral boundary of $\mathcal{S}_{T}$.

With $\|v\|_{L^{r}\left(\mathcal{S}_{T}\right)}$ we will always denote the norm of $v \in L^{r}\left(\mathcal{S}_{T}\right)$ and with $|u|_{L^{r}(0, T)^{N}}$ the $L^{r}(0, T)^{N}$ norm of $u \in L^{r}(0, T)^{N}$.

Let $\mathcal{C}_{T}^{\infty}\left(\mathbb{R}^{N}\right)$ be the space of functions $u \in \mathcal{C}^{\infty}\left(\mathbb{R}^{N}\right)$ such that $u$ is $T$-periodic in each variable, that is

$$
u\left(x+T e_{i}\right)=u(x) \text { for all } x \in \mathbb{R}^{N}, i \in \mathbb{Z}[1, N] .
$$

We define the fractional Sobolev space $\mathbb{H}_{T}^{s}$ as the closure of $\mathcal{C}_{T}^{\infty}\left(\mathbb{R}^{N}\right)$ endowed by the norm

$$
|u|_{\mathbb{H}_{T}^{s}}:=\sqrt{\sum_{k \in \mathbb{Z}^{N}}\left(\omega^{2}|k|^{2}+m^{2}\right)^{s}\left|\beta_{k}\right|^{2}}
$$


Let us introduce the functional space $\mathbb{X}_{T}^{s}$ defined as the completion of

$$
\begin{array}{r}
\mathcal{C}_{T}^{\infty}\left(\overline{\mathbb{R}_{+}^{N+1}}\right):=\left\{v \in \mathcal{C}^{\infty}\left(\overline{\mathbb{R}_{+}^{N+1}}\right): v\left(x+T e_{i}, y\right)=v(x, y)\right. \\
\left.\quad \text { for every }(x, y) \in \overline{\mathbb{R}_{+}^{N+1}}, i=1, \ldots, N\right\}
\end{array}
$$

under the $H^{1}\left(\mathcal{S}_{T}, y^{1-2 s}\right)$ norm

$$
\|v\|_{\mathbb{X}_{T}^{s}}:=\sqrt{\iint_{\mathcal{S}_{T}} y^{1-2 s}\left(|\nabla v|^{2}+m^{2} v^{2}\right) d x d y} .
$$

We recall that it is possible to define a trace operator between $\mathbb{X}_{T}^{s}$ and $\mathbb{H}_{T}^{s}$ (see $[2,3]$ for details):

Theorem 3. There exists a surjective linear operator $\operatorname{Tr}: \mathbb{X}_{T}^{s} \rightarrow \mathbb{H}_{T}^{s}$ such that:

(i) $\operatorname{Tr}(v)=\left.v\right|_{\partial^{0} \mathcal{S}_{T}}$ for all $v \in \mathcal{C}_{T}^{\infty}\left(\overline{\mathbb{R}_{+}^{N+1}}\right) \cap \mathbb{X}_{T}^{s} ;$

(ii) $\operatorname{Tr}$ is bounded and

$$
\sqrt{\kappa_{s}}|\operatorname{Tr}(v)|_{\mathbb{H}_{T}^{s}} \leqslant\|v\|_{\mathbb{X}_{T}^{s}}
$$

for every $v \in \mathbb{X}_{T}^{s}$. In particular, equality holds in (2.1) for some $v \in \mathbb{X}_{T}^{s}$ if and only if $v$ weakly solves the following equation

$$
-\operatorname{div}\left(y^{1-2 s} \nabla v\right)+m^{2} y^{1-2 s} v=0 \text { in } \mathcal{S}_{T} .
$$

Moreover, we have the following embedding results:

Theorem 4. Let $N \geqslant 2$ and $s \in(0,1)$. Then $\operatorname{Tr}\left(\mathbb{X}_{T}^{s}\right)$ is continuously embedded in $L^{q}(0, T)^{N}$ for any $1 \leqslant q \leqslant 2_{s}^{\sharp}$. Moreover, $\operatorname{Tr}\left(\mathbb{X}_{T}^{s}\right)$ is compactly embedded in $L^{q}(0, T)^{N}$ for any $1 \leqslant q<2_{s}^{\sharp}$.

Now, we aim to reformulate the nonlocal problem (1.2) in a local way. Let $g \in \mathbb{H}_{T}^{-s}$, where

$$
\mathbb{H}_{T}^{-s}:=\left\{g=\sum_{k \in \mathbb{Z}^{N}} g_{k} \frac{e^{\imath \omega k \cdot x}}{\sqrt{T^{N}}}: \sum_{k \in \mathbb{Z}^{N}} \frac{\left|g_{k}\right|^{2}}{\left(\omega^{2}|k|^{2}+m^{2}\right)^{s}}<+\infty\right\}
$$

is the dual of $\mathbb{H}_{T}^{s}$, and consider the following two problems:

$$
\begin{cases}\left(-\Delta+m^{2}\right)^{s} u=g & \text { in }(0, T)^{N} \\ u\left(x+T e_{i}\right)=u(x) & \text { for all } x \in \mathbb{R}^{N}\end{cases}
$$

and

$$
\begin{cases}-\operatorname{div}\left(y^{1-2 s} \nabla v\right)+m^{2} y^{1-2 s} v=0 & \text { in } \mathcal{S}_{T} \\ v_{\mid\left\{x_{i}=0\right\}}=v_{\mid\left\{x_{i}=T\right\}} & \text { on } \partial_{L} \mathcal{S}_{T} \\ \partial_{\nu}^{1-2 s} v=\kappa_{s} g(x) & \text { on } \partial^{0} \mathcal{S}_{T}\end{cases}
$$

Then we have the following definitions of weak solutions to (2.3) and (2.2) respectively: 
Definition 1. We say that $v \in \mathbb{X}_{T}^{s}$ is a weak solution to (2.3) if for every $\varphi \in \mathbb{X}_{T}^{s}$ it holds

$$
\iint_{\mathcal{S}_{T}} y^{1-2 s}\left(\nabla v \nabla \varphi+m^{2} v \varphi\right) d x d y=\kappa_{s}\langle g, \operatorname{Tr}(\varphi)\rangle .
$$

Here $\langle\cdot, \cdot\rangle$ is the duality pairing between $\mathbb{H}_{T}^{s}$ and its dual $\mathbb{H}_{T}^{-s}$.

Definition 2. We say that $u \in \mathbb{H}_{T}^{s}$ is a weak solution to (2.2) if $u=$ $\operatorname{Tr}(v)$ and $v \in \mathbb{X}_{T}^{s}$ is a weak solution to (2.3).

Taking into account Theorem 3 and Theorem 4, it is possible to introduce the notion of extension for a function $u \in \mathbb{H}_{T}^{s}$.

More precisely, the next result holds.

Theorem 5. Let $u \in \mathbb{H}_{T}^{s}$. Then, there exists a unique $v \in \mathbb{X}_{T}^{s}$ such that

$$
\begin{cases}-\operatorname{div}\left(y^{1-2 s} \nabla v\right)+m^{2} y^{1-2 s} v=0 & \text { in } \mathcal{S}_{T} \\ v_{\mid\left\{x_{i}=0\right\}}=v_{\mid\left\{x_{i}=T\right\}} & \text { on } \partial_{L} \mathcal{S}_{T} \\ v(\cdot, 0)=u & \text { on } \partial^{0} \mathcal{S}_{T}\end{cases}
$$

and

$$
-\lim _{y \rightarrow 0^{+}} y^{1-2 s} \frac{\partial v}{\partial y}(x, y)=\kappa_{s}\left(-\Delta+m^{2}\right)^{s} u(x) \text { in } \mathbb{H}_{T}^{-s} .
$$

We call $v \in \mathbb{X}_{T}^{s}$ the extension of $u \in \mathbb{H}_{T}^{s}$.

In particular, if $u=\sum_{k \in \mathbb{Z}^{N}} \beta_{k} \frac{e^{\imath \omega k \cdot x}}{\sqrt{T^{N}}}$, then $v$ is given by

$$
v(x, y)=\sum_{k \in \mathbb{Z}^{N}} \beta_{k} \theta_{k}(y) \frac{e^{\imath \omega k \cdot x}}{\sqrt{T^{N}}}
$$

where $\theta_{k}(y):=\theta\left(\sqrt{\omega^{2}|k|^{2}+m^{2}} y\right)$ and $\theta(y) \in H^{1}\left(\mathbb{R}_{+}, y^{1-2 s}\right)$ solves the following $O D E$

$$
\left\{\begin{array}{c}
\theta^{\prime \prime}+\frac{1-2 s}{y} \theta^{\prime}-\theta=0 \text { in } \mathbb{R}_{+} . \\
\theta(0)=1 \text { and } \theta(\infty)=0
\end{array} .\right.
$$

Moreover, $v$ satisfies the properties:

(i) $v$ is smooth for $y>0$ and $T$-periodic in $x$;

(ii) $\|v\|_{\mathbb{X}_{T}^{s}} \leqslant\|z\|_{\mathbb{X}_{T}^{s}}$ for any $z \in \mathbb{X}_{T}^{s}$ such that $\operatorname{Tr}(z)=u$;

(iii) $\|v\|_{\mathbb{X}_{T}^{s}}=\sqrt{\kappa_{s}}|u|_{\mathbb{H}_{T}^{s}}$.

Now, we are in the position to reformulate the nonlocal problem (1.2) with periodic boundary conditions, in a local way according the following definitions. 
Definition 3. We say that $v \in \mathbb{X}_{T}^{s}$ is a weak solution to (1.5) if

$$
\begin{gathered}
\iint_{\mathcal{S}_{T}} y^{1-2 s}\left(\nabla v \nabla \varphi+m^{2} v \varphi\right) d x d y=\kappa_{s} \gamma_{\infty} \int_{\partial^{0} \mathcal{S}_{T}} \operatorname{Tr}(v) \operatorname{Tr}(\varphi) d x \\
+\lambda \kappa_{s} \int_{\partial^{0} \mathcal{S}_{T}} f(x, \operatorname{Tr}(v)) \operatorname{Tr}(\varphi) d x
\end{gathered}
$$

for every $\varphi \in \mathbb{X}_{T}^{s}$.

Finally, we give the notion of weak solution to problem (1.2).

Definition 4. We say that $u \in \mathbb{H}_{T}^{s}$ is a weak solution to (1.2) if $u=$ $\operatorname{Tr}(v)$ and $v \in \mathbb{X}_{T}^{s}$ is a weak solution to (1.5).

2.2. On periodic eigenvalue problem in the half-cylinder. The study of the eigenvalues of a linear operator is a classical topic and many functional analytic tools of general flavor may be used to deal with it. The result that we give here is, indeed, more general and more precise than what we need, strictly speaking, for the proofs of our main results: nevertheless we believed it was good to have a result stated in detail also for further reference. Hence, we focus on the following eigenvalue problem

$$
\left\{\begin{array}{ll}
\left(-\Delta+m^{2}\right)^{s} u=\lambda u & \text { in }(0, T)^{N} \\
u\left(x+T e_{i}\right)=u(x) & \forall x \in \mathbb{R}^{N}, i \in \mathbb{Z}[1, N]
\end{array} .\right.
$$

More precisely, we discuss the weak formulation of (2.5), which consists in the following eigenvalue problem:

Find $u \in \mathbb{H}_{T}^{s}$ such that $u=\operatorname{Tr}(v)$ where $v \in \mathbb{X}_{T}^{s}$ and

$$
\begin{array}{r}
\iint_{\mathcal{S}_{T}} y^{1-2 s}\left(\nabla v \nabla \varphi+m^{2} v \varphi\right) d x d y \\
=\lambda \kappa_{s} \int_{\partial^{0} \mathcal{S}_{T}} \operatorname{Tr}(v) \operatorname{Tr}(\varphi) d x
\end{array}
$$

for every $\varphi \in \mathbb{X}_{T}^{s}$.

In other words, $v \in \mathbb{X}_{T}^{s}$ is a weak solution of the extended problem

$$
\left\{\begin{array}{ll}
-\operatorname{div}\left(y^{1-2 s} \nabla v\right)+m^{2} y^{1-2 s} v=0 & \text { in } \mathcal{S}_{T} \\
v_{\mid\left\{x_{i}=0\right\}}=v_{\mid\left\{x_{i}=T\right\}} & \text { on } \partial_{L} \mathcal{S}_{T} \\
-\lim _{y \rightarrow 0^{+}} y^{1-2 s} \frac{\partial v}{\partial y}(x, y)=\lambda \kappa_{s} v & \text { on } \partial^{0} \mathcal{S}_{T}
\end{array} .\right.
$$

We recall that $\lambda \in \mathbb{R}$ is an eigenvalue of $\left(-\Delta+m^{2}\right)^{s}$ provided there exists a non-trivial weak solution of (2.5).

Following the classical spectral theory [33, 41], the powers of a positive operator in a bounded domain are defined through the spectral 
decomposition using the powers of the eigenvalues of the original operator.

Then, we can derive the following result:

Lemma 1. With the above notations the following facts hold:

(i) The operator $\left(-\Delta+m^{2}\right)^{s}$ has a countable family of eigenvalues $\left\{\lambda_{\ell}\right\}_{\ell \in \mathbb{N}}$ which can be written as an increasing sequence of positive numbers

$$
0<\lambda_{1}<\lambda_{2} \leqslant \ldots \leqslant \lambda_{\ell} \leqslant \lambda_{\ell+1} \leqslant \ldots
$$

Each eigenvalue is repeated a number of times equal to its multiplicity (which is finite);

(ii) $\lambda_{\ell}=\mu_{\ell}^{s}$ for all $\ell \in \mathbb{N}$, where $\left\{\mu_{\ell}\right\}_{\ell \in \mathbb{N}}$ is the increasing sequence of eigenvalues of $-\Delta+m^{2}$;

(iii) $\lambda_{1}=m^{2 s}$ is simple, $\lambda_{\ell}=\mu_{\ell}^{s} \rightarrow+\infty$ as $\ell \rightarrow+\infty$;

(iv) The sequence $\left\{u_{\ell}\right\}_{\ell \in \mathbb{N}}$ of eigenfunctions corresponding to $\lambda_{\ell}$ is an orthonormal basis of $L^{2}(0, T)^{N}$ and an orthogonal basis of the Sobolevspace $\mathbb{H}_{T}^{s}$. Let us note that $\left\{u_{\ell}, \mu_{\ell}\right\}_{\ell \in \mathbb{N}}$ are the eigenfunctions and eigenvalues of $-\Delta+m^{2}$ under periodic boundary conditions;

(v) For any $h \in \mathbb{N}$, $\lambda_{\ell}$ has finite multiplicity, and there holds

$$
\lambda_{\ell}=\min _{u \in \mathbb{P}_{\ell} \backslash\{0\}} \frac{|u|_{\mathbb{H}_{T}^{s}}^{2}}{|u|_{L^{2}(0, T)^{N}}^{2}} \quad \text { (Rayleigh's principle) }
$$

where

$$
\mathbb{P}_{\ell}:=\left\{u \in \mathbb{H}_{T}^{s}:\left\langle u, u_{j}\right\rangle_{\mathbb{H}_{T}^{s}}=0, \text { for } j=1, \ldots, \ell-1\right\} .
$$

Proof. It is enough to prove that $\left(-\Delta+m^{2}\right)^{-s}: L^{2}(0, T)^{N} \rightarrow L^{2}(0, T)^{N}$ is a self-adjoint, positive and compact operator.

Firstly we observe that if $u=\sum_{k \in \mathbb{Z}^{N}} c_{k} e^{\imath \omega k \cdot x}$ and $v=\sum_{k \in \mathbb{Z}^{N}} d_{k} e^{\imath \omega k \cdot x}$ belong to $L^{2}(0, T)^{N}$, then

$$
\begin{aligned}
\left\langle\left(-\Delta+m^{2}\right)^{-s} u, v\right\rangle_{L^{2}(0, T)^{N}} & =\sum_{k \in \mathbb{Z}^{N}} \frac{c_{k}}{\left(\omega^{2}|k|^{2}+m^{2}\right)^{s}} \bar{d}_{k} \\
& =\sum_{k \in \mathbb{Z}^{N}} c_{k} \frac{\bar{d}_{k}}{\left(\omega^{2}|k|^{2}+m^{2}\right)^{s}} \\
& =\left\langle u,\left(-\Delta+m^{2}\right)^{-s} v\right\rangle_{L^{2}(0, T)^{N}}
\end{aligned}
$$


that is $\left(-\Delta+m^{2}\right)^{-s}$ is self-adjoint.

Clearly, for $u=\sum_{k \in \mathbb{Z}^{N}} c_{k} e^{\imath \omega k \cdot x} \in L^{2}(0, T)^{N}$ we have

$$
\left\langle\left(-\Delta+m^{2}\right)^{-s} u, u\right\rangle_{L^{2}(0, T)^{N}}=\sum_{k \in \mathbb{Z}^{N}} \frac{\left|c_{k}\right|^{2}}{\left(\omega^{2}|k|^{2}+m^{2}\right)^{s}} \geqslant 0
$$

and $\left\langle\left(-\Delta+m^{2}\right)^{-s} u, u\right\rangle_{L^{2}(0, T)^{N}}>0$ if $u \neq 0$.

Finally, we show that $\left(-\Delta+m^{2}\right)^{-s}$ is compact. Let $\left\{v_{j}\right\}_{j \in \mathbb{N}}$ be a bounded sequence in $L^{2}(0, T)^{N}$ and let us denote by $\left\{d_{k}^{j}\right\}_{k \in \mathbb{Z}^{N}}$ its Fourier coefficients.. Since $L^{2}(0, T)^{N} \subset \mathbb{H}_{T}^{-s}$, it is clear that $\left\{v_{j}\right\}_{j \in \mathbb{N}}$ is bounded in $\mathbb{H}_{T}^{-s}$. Let us denote by $u_{j}=\left(-\Delta+m^{2}\right)^{-s} v_{j}$.

Then

$$
\left|u_{j}\right|_{\mathbb{H}_{T}^{s}}^{2}=\sum_{k \in \mathbb{Z}^{N}}\left(\omega^{2}|k|^{2}+m^{2}\right)^{s} \frac{\left|d_{k}^{j}\right|^{2}}{\left(\omega^{2}|k|^{2}+m^{2}\right)^{2 s}}=\left|v_{j}\right|_{\mathbb{H}_{T}^{-s}}^{2},
$$

that is $\left\{u_{j}\right\}_{j \in \mathbb{N}}$ is a bounded sequence in $\mathbb{H}_{T}^{s}$. By using the compactness of $\mathbb{H}_{T}^{s}$ into $L^{2}(0, T)^{N}$, we deduce that $\left\{u_{j}\right\}_{j \in \mathbb{N}}$ admits a convergent subsequence in $L^{2}(0, T)^{N}$. As a consequence $\left(-\Delta+m^{2}\right)^{-s} v_{j}$ strongly converges in $L^{2}(0, T)^{N}$.

Now, we aim to find some useful relation between the eigenvalues $\left\{\lambda_{j}\right\}_{j \in \mathbb{N}}$ of $\left(-\Delta+m^{2}\right)^{s}$ and the corresponding extended eigenvalue problem in the half-cylinder $\mathcal{S}_{T}$,

$$
\begin{cases}-\operatorname{div}\left(y^{1-2 s} \nabla v\right)+m^{2} y^{1-2 s} v=0 & \text { in } \mathcal{S}_{T} \\ v_{\mid\left\{x_{i}=0\right\}}=v_{\mid\left\{x_{i}=T\right\}} & \text { on } \partial_{L} \mathcal{S}_{T} . \\ \partial_{\nu}^{1-2 s} v=\lambda_{j} \kappa_{s} v & \text { on } \partial^{0} \mathcal{S}_{T}\end{cases}
$$

Let us introduce the following notations. Set

$$
\mathbb{V}_{h}:=\operatorname{Span}\left\{v_{1}, \ldots, v_{h}\right\},
$$

where every $v_{j}$ solves (2.7). Clearly $\operatorname{Tr}\left(v_{j}\right)=u_{j}$ for all $j \in \mathbb{N}$, where $\left\{u_{j}\right\}_{j \in \mathbb{N}}$ is the basis of eigenfunctions in $\mathbb{H}_{T}^{s}$, defined in Lemma 1. For any $h \in \mathbb{N}$ we define

$$
\mathbb{V}_{h}^{\perp}:=\left\{v \in \mathbb{X}_{T}^{s}:\left\langle v, v_{j}\right\rangle_{\mathbb{X}_{T}^{s}}=0, \text { for } j=1, \ldots, h\right\} .
$$

Since $v_{j}$ solves (2.7), then we deduce that

$$
\mathbb{V}_{h}^{\perp}=\left\{v \in \mathbb{X}_{T}^{s}:\left\langle\operatorname{Tr}(v), \operatorname{Tr}\left(v_{j}\right)\right\rangle_{L^{2}(0, T)^{N}}=0, \text { for } j=1, \ldots, h\right\} .
$$

Then $\mathbb{X}_{T}^{s}=\mathbb{V}_{h} \bigoplus \mathbb{V}_{h}^{\perp}$. Let us point out that the trace operator is bijective on $E:=\left\{v \in \mathbb{X}_{T}^{s}: v\right.$ solves $\left.(2.4)\right\}$.

Indeed, if $\tilde{v}_{1}$ and $\tilde{v}_{2}$ are the extension of $\tilde{u}_{1}, \tilde{u}_{2} \in \mathbb{H}_{T}^{s}$ respectively, then

$$
\left\langle\tilde{v}_{i}, \varphi\right\rangle_{\mathbb{X}_{T}^{s}}=k_{s}\left\langle\tilde{u}_{i}, \operatorname{Tr}(\varphi)\right\rangle_{\mathbb{H}_{T}^{s}} \quad \forall \varphi \in \mathbb{X}_{T}^{s}, i=1,2
$$


If $\tilde{u}_{1}=\operatorname{Tr}\left(\tilde{v}_{1}\right)=\operatorname{Tr}\left(\tilde{v}_{2}\right)=\tilde{u}_{2}$, from (2.10) follows that

$$
\left\langle\tilde{v}_{1}-\tilde{v}_{2}, \varphi\right\rangle_{\mathbb{X}_{T}^{s}}=0 \quad \forall \varphi \in \mathbb{X}_{T}^{s},
$$

so we deduce that $\tilde{v}_{1}=\tilde{v}_{2}$, that is $\operatorname{Tr}$ is injective on $E$.

From this and the linearity of the trace operator Tr, we have

$$
\operatorname{dim} \mathbb{V}_{h}=\operatorname{dim} \operatorname{Span}\left\{\operatorname{Tr}\left(v_{1}\right), \cdots, \operatorname{Tr}\left(v_{h}\right)\right\}=h .
$$

Now we prove that $\|\cdot\|_{\mathbb{X}_{T}^{s}}$ and $|\cdot|_{2}$ are equivalent norms on the finite dimensional space $\mathbb{V}_{h}$.

More precisely, for any $v \in \mathbb{V}_{h}$, it results

$$
\kappa_{s} m^{2 s}|\operatorname{Tr}(v)|_{2}^{2} \leqslant\|v\|_{\mathbb{X}^{s}}^{2} \leqslant \kappa_{s} \lambda_{h}|\operatorname{Tr}(v)|_{2}^{2} .
$$

Firstly we note that $\left\{v_{j}\right\}_{j \in \mathbb{N}}$ is an orthogonal system in $\mathbb{X}_{T}^{s}$, since $\left\{\operatorname{Tr}\left(v_{j}\right)\right\}_{j \in \mathbb{N}}$ is an orthonormal system in $L^{2}(0, T)^{N}$, and $v_{j}$ satisfies

$$
\left\langle z, v_{j}\right\rangle_{\mathbb{X}_{T}^{s}}=\kappa_{s} \lambda_{j}\left\langle\operatorname{Tr}(z), \operatorname{Tr}\left(v_{j}\right)\right\rangle_{L^{2}(0, T)^{N}} \text { for all } z \in \mathbb{X}^{s}, j \in \mathbb{N} .
$$

Then, by using the fact that $\left\{\lambda_{j}\right\}_{j \in \mathbb{N}}$ is an increasing sequence (see (i) of Lemma 1), and by trace inequality (2.1), for $v=\sum_{j=1}^{h} \alpha_{j} v_{j} \in \mathbb{V}_{h}$ we have

$$
\begin{aligned}
\kappa_{s} m^{2 s}|\operatorname{Tr}(v)|_{2}^{2} & \leqslant\|v\|_{\mathbb{X}^{s}}^{2}=\sum_{j=1}^{h} \alpha_{j}^{2}\left\|v_{j}\right\|_{\mathbb{X}^{s}}^{2} \\
& =\kappa_{s} \sum_{j=1}^{h} \lambda_{j} \alpha_{j}^{2}\left|\operatorname{Tr}\left(v_{j}\right)\right|_{2}^{2} \leqslant \kappa_{s} \lambda_{h} \sum_{j=1}^{h} \alpha_{j}^{2}\left|\operatorname{Tr}\left(v_{j}\right)\right|_{2}^{2} \\
& =\kappa_{s} \lambda_{h}|\operatorname{Tr}(v)|_{2}^{2} .
\end{aligned}
$$

Finally we prove that for any $v \in \mathbb{V}_{h}^{\perp}$ the following inequality holds

$$
\lambda_{h+1}|\operatorname{Tr}(v)|_{2}^{2} \leqslant \frac{1}{\kappa_{s}}\|v\|_{\mathbb{X}_{T}^{s}}^{2} .
$$

Fix $v \in \mathbb{V}_{h}^{\perp}$. Then $\operatorname{Tr}(v) \in \mathbb{P}_{h+1}$. Indeed $\operatorname{Tr}\left(v_{j}\right)=u_{j}$ is a weak solution to $\left(-\Delta+m^{2}\right)^{s} u=\lambda_{j} u$ and by using the fact that

$$
\left\langle\operatorname{Tr}(v), \operatorname{Tr}\left(v_{j}\right)\right\rangle_{L^{2}(0, T)^{N}}=0 \text { for every } j=1, \ldots, h,
$$

we can infer that $\left\langle\operatorname{Tr}(v), \operatorname{Tr}\left(v_{j}\right)\right\rangle_{\mathbb{H}_{T}^{s}}=0$, for every $j=1, \ldots, h$.

As a consequence, by using the variational characterization $(v)$ of Lemma 1 and the trace inequality (2.1), we get

$$
\lambda_{h+1}|\operatorname{Tr}(v)|_{2}^{2} \leqslant|\operatorname{Tr}(v)|_{\mathbb{H}_{T}^{s}}^{2} \leqslant \frac{1}{\kappa_{s}}\|v\|_{\mathbb{X}_{T}^{s}}^{2}
$$


2.3. An abstract critical point theorem. Since (1.5) has a variational structure, in this section we provide the main classical tools that we use for its study.

Let us denote by $\left(E,\|\cdot\|_{E}\right)$ a Banach space, $\left(E^{\prime},\|\cdot\|_{E^{\prime}}\right)$ its dual, $\Phi$ a $C^{1}$ functional on $E, \Phi^{b}:=\{e \in E: \Phi(e) \leqslant b\}$ the sublevel of $\Phi$ corresponding to $b \in \overline{\mathbb{R}}:=\mathbb{R} \cup\{ \pm \infty\}$ and by

$$
K_{c}:=\{e \in E: \Phi(e)=c, \mathrm{~d} \Phi(e)=0\}
$$

the set of the critical points of $\Phi$ in $E$ at the critical level $c \in \mathbb{R}$.

The functional $\Phi$ satisfies the Palais-Smale condition, briefly (PS), at level $c(c \in \mathbb{R})$, if any sequence $\left\{u_{j}\right\}_{j \in \mathbb{N}} \subseteq E$ such that

$$
\lim _{j \rightarrow+\infty} \Phi\left(u_{j}\right)=c \quad \text { and } \quad \lim _{j \rightarrow+\infty}\left\|\mathrm{d} \Phi\left(u_{j}\right)\right\|_{E^{\prime}}=0
$$

converges in $E$, up to subsequences. In general, if $-\infty \leqslant a<b \leqslant+\infty$, $\Phi$ satisfies (PS) in $(a, b)$ if so is at each level $c \in(a, b)$.

As usual, the classical Ambrosetti-Rabinowitz condition plays a crucial role in proving that every Palais-Smale sequence is bounded, as well as the so called mountain-pass geometry is satisfied. However, even dealing with different problems than ours, several authors studied different assumptions that still allow to apply min-max procedure in order to assure the existence of critical points. For asymptotically linear problems we show a result that moves along this direction.

Let us recall some basic notions of the index theory for an even functional with symmetry group $\mathbb{Z}_{2}=\{\mathrm{id},-\mathrm{id}\}$. Let us set

$$
\begin{aligned}
\Sigma:=\{A \subseteq E: & A \text { closed and symmetric w.r.t. the origin, } \\
& \text { i.e. }-e \in A \text { if } e \in A\}
\end{aligned}
$$

and

$$
\mathcal{H}:=\{h \in C(E, E): h \text { odd }\} .
$$

For $A \in \Sigma, A \neq \emptyset$, the genus of $A$ is

$$
\gamma(A):=\inf \left\{m \in \mathbb{N}: \exists \psi \in C\left(A, \mathbb{R}^{m} \backslash\{0\}\right) \text { s.t. } \psi(-e)=-\psi(e), \forall e \in A\right\}
$$

if such an infimum exists, otherwise $\gamma(A)=+\infty$. Assume $\gamma(\emptyset)=0$.

The index theory $(\Sigma, \mathcal{H}, \gamma)$ related to $\mathbb{Z}_{2}$ is also called genus (we refer for more details to [39, Section II.5]).

The pseudo-index related to the genus and $S \in \Sigma$ is the triplet $\left(S, \mathcal{H}^{*}, \gamma^{*}\right)$ such that $\mathcal{H}^{*}$ is a group of odd homeomorphisms and $\gamma^{*}$ : $\Sigma \longrightarrow \mathbb{N} \cup\{+\infty\}$ is the map defined by

$$
\gamma^{*}(A):=\min _{h \in \mathcal{H}^{*}} \gamma(h(A) \cap S), \quad \forall A \in \Sigma
$$

(cf. [14] for more details). 
The proof of our main multiplicity result, Theorem 2, is based on the following abstract result proved in [9, Theorem 2.9].

Theorem 6. Let $a, b, c_{0}, c_{\infty} \in \overline{\mathbb{R}},-\infty \leqslant a<c_{0}<c_{\infty}<b \leqslant+\infty$, $\Phi$ be an even functional, $(\Sigma, \mathcal{H}, \gamma)$ the genus theory on $E, S \in \Sigma,\left(S, \mathcal{H}^{*}, \gamma^{*}\right)$ the pseudo-index theory related to the genus and $S$, with

$$
\begin{array}{r}
\mathcal{H}^{*}:=\{h \in \mathcal{H}: h \text { bounded homeomorphism } \\
\text { such that } \left.h(e)=e \text { if } e \notin \Phi^{-1}((a, b))\right\} .
\end{array}
$$

Assume that:

(i) the functional $\Phi$ satisfies (PS) in $(a, b)$;

(ii) $S \subseteq \Phi^{-1}\left(\left[c_{0},+\infty\right)\right)$;

(iii) there exist $\tilde{k} \in \mathbb{N}$ and $\tilde{A} \in \Sigma$ such that $\tilde{A} \subseteq \Phi^{c_{\infty}}$ and $\gamma^{*}(\tilde{A}) \geqslant \tilde{k}$. Then, setting $\Sigma_{i}^{*}:=\left\{A \in \Sigma: \gamma^{*}(A) \geqslant i\right\}$, the numbers

$$
c_{i}:=\inf _{A \in \Sigma_{i}^{*}} \sup _{e \in A} \Phi(e), \quad \forall i \in\{1, \ldots, \tilde{k}\},
$$

are critical values for $\Phi$ and

$$
c_{0} \leqslant c_{1} \leqslant \ldots \leqslant c_{\tilde{k}} \leqslant c_{\infty}
$$

Furthermore, if $c=c_{i}=\ldots=c_{i+r}$, with $i \geqslant 1$ and $i+r \leqslant \tilde{k}$, then $\gamma\left(K_{c}\right) \geqslant r+1$.

Remark 1. If $\Phi \in C^{1}(E, \mathbb{R})$ and $E$ is a Hilbert space, by Riesz theorem there exists a unique $\Phi^{\prime}(u) \in E$ such that

$$
\left\langle\Phi^{\prime}(u), v\right\rangle=\mathrm{d} \Phi(u)[v] \quad \forall v \in E .
$$

$\Phi^{\prime}(u)$ is called the gradient of $\Phi$ in $u$. With this notation, a critical point of $\Phi$ is a solution to $\Phi^{\prime}(u)=0$.

Remark 2. In the applications, a lower bound for the pseudo-index of a suitable $\tilde{A}$ as in (iii) of Theorem 6 is needed: considering the genus theory $(\Sigma, \mathcal{H}, \gamma)$ on $E$ and $V, W$ two closed subspaces of $E$, if

$$
\operatorname{dim} V<+\infty \text { and } \operatorname{codim} W<+\infty,
$$

then

$$
\gamma(V \cap h(\partial B \cap W)) \geqslant \operatorname{dim} V-\operatorname{codim} W
$$

for every bounded $h \in \mathcal{H}$ and every open bounded symmetric neighbourhood $B$ of 0 in $E$ (cf. [9, Theorem A.2]). 


\section{PERIODIC SOLUTIONS VIA SADDLE POINT THEOREM}

In order to obtain weak solutions of problem (1.5), we study the critical points of the following functional

$$
\mathcal{J}(v):=\frac{1}{2}\|v\|_{\mathbb{X}_{T}^{s}}^{2}-\frac{\lambda_{\infty} \kappa_{s}}{2}|\operatorname{Tr}(v)|_{L^{2}(0, T)^{N}}^{2} d x-\kappa_{s} \int_{\partial^{0} \mathcal{S}_{T}} F(x, \operatorname{Tr}(v)) d x
$$

defined on the Hilbert space $\mathbb{X}_{T}^{s}$ and where, as usual, we set

$$
F(x, t):=\int_{0}^{t} f(x, \tau) d \tau .
$$

Now, we prove that the functional $\mathcal{J}$ is smooth and it has the geometric structure required by the Saddle Point Theorem (see, for instance, [32, Theorem 4.6]).

Here, we use the structural assumptions on $f$ to deduce some bounds from above and below for the nonlinear term and its primitive. This part is quite standard and does not take into account the nonlocal features of the problem.

By the growth condition $\left(f_{1}\right)$ and (1.3) for all $\varepsilon>0$ there exists $a_{\varepsilon}>0$ such that

$$
|f(x, t)| \leqslant \varepsilon|t|+a_{\varepsilon}, \quad \text { for a.e. } x \in(0, T)^{N}, \forall t \in \mathbb{R} .
$$

so, in particular, we deduce that

$$
|F(x, t)| \leqslant c_{1}\left(1+t^{2}\right), \quad \text { for a.e. } x \in(0, T)^{N}, \forall t \in \mathbb{R} .
$$

Then, by using Theorem 4, it follows that the functional $\mathcal{J}$ is welldefined and $\mathcal{J} \in C^{1}\left(\mathbb{X}_{T}^{s}, \mathbb{R}\right)$. In particular, its gradient is given by

$$
\begin{aligned}
\left\langle\mathcal{J}^{\prime}(v), \varphi\right\rangle & =\iint_{\mathcal{S}_{T}} y^{1-2 s}\left(\nabla v \nabla \varphi+m^{2} v \varphi\right) d x d y \\
& -\lambda_{\infty} \kappa_{s} \int_{\partial^{0} \mathcal{S}_{T}} \operatorname{Tr}(v) \operatorname{Tr}(\varphi) d x-\kappa_{s} \int_{\partial^{0} \mathcal{S}_{T}} f(x, \operatorname{Tr}(v)) \operatorname{Tr}(\varphi) d x
\end{aligned}
$$

for every $\varphi \in \mathbb{X}_{T}^{s}$.

Therefore, in order to apply critical point methods, we have to check the validity of the Palais-Smale condition, that is

$$
\begin{gathered}
\text { for any } c \in \mathbb{R} \text { any sequence }\left\{v_{j}\right\}_{j \in \mathbb{N}} \text { in } \mathbb{X}_{T}^{s} \text { such that } \\
\mathcal{J}\left(v_{j}\right) \rightarrow c \text { and } \sup \left\{\left|\left\langle\mathcal{J}^{\prime}\left(v_{j}\right), \varphi\right\rangle\right|: \varphi \in \mathbb{X}_{T}^{s},\|\varphi\|_{\mathbb{X}_{T}^{s}}=1\right\} \rightarrow 0 \\
\text { as } j \rightarrow+\infty \text {, admits a subsequence strongly convergent in } \mathbb{X}_{T}^{s} .
\end{gathered}
$$

Hence, in the next proposition we prove that in the non-resonant case the functional $\mathcal{J}$ satisfies the (PS) condition. 
Proposition 1. Assume that $\left(f_{1}\right)$ and (1.3) of $\left(f_{2}\right)$ hold. Then, if $\lambda_{\infty} \notin \sigma\left(\left(-\Delta+m^{2}\right)^{s}\right)$, the functional $\mathcal{J}$ verifies the (PS) in $\mathbb{R}$.

Proof. Let $c \in \mathbb{R}$ and $\left\{v_{j}\right\}_{j \in \mathbb{N}}$ be a sequence in $\mathbb{X}_{T}^{s}$ such that

$$
\mathcal{J}\left(v_{j}\right) \rightarrow c \text { and }\left\|\mathcal{J}^{\prime}\left(v_{j}\right)\right\|_{\mathbb{X}_{T}^{-s}} \rightarrow 0 \text { as } j \rightarrow \infty,
$$

where $\mathbb{X}_{T}^{-s}$ is the dual space of $\mathbb{X}_{T}^{s}$. Hence, it follows that

$$
\begin{aligned}
\left\langle v_{j}, \varphi\right\rangle_{\mathbb{X}_{T}^{s}} & -\lambda_{\infty} \kappa_{s} \int_{\partial^{0} \mathcal{S}_{T}} \operatorname{Tr}\left(v_{j}\right) \operatorname{Tr}(\varphi) d x \\
& -\kappa_{s} \int_{\partial^{0} \mathcal{S}_{T}} f\left(x, \operatorname{Tr}\left(v_{j}\right)\right) \operatorname{Tr}(\varphi) d x=o(1)
\end{aligned}
$$

for all $\varphi \in \mathbb{X}_{T}^{s}$, where $o(1)$ denotes an infinitesimal sequence.

First of all, as usual when using variational methods, we prove the boundedness of a Palais-Smale sequence for $\mathcal{J}$. Hence, we prove that $\left\{v_{j}\right\}_{j \in \mathbb{N}}$ is bounded in $\mathbb{X}_{T}^{s}$. Let us assume by contradiction that

$$
\left\|v_{j}\right\|_{\mathbb{X}_{T}^{s}} \rightarrow+\infty \quad \text { as } j \rightarrow+\infty \text {. }
$$

By setting $w_{j}:=\frac{v_{j}}{\left\|v_{j}\right\|_{\mathbb{X}_{T}^{s}}}$, it is clear that $\left\{w_{j}\right\}_{j \in \mathbb{N}}$ is bounded in $\mathbb{X}_{T}^{s}$ and by using Theorem 3 , there exists $w \in \mathbb{X}_{T}^{s}$ such that, up to subsequences, it results

$$
\begin{aligned}
& w_{j} \rightarrow w \quad \text { weakly in } \mathbb{X}_{T}^{s} \\
& \operatorname{Tr}\left(w_{j}\right) \rightarrow \operatorname{Tr}(w) \quad \text { strongly in } L^{2}(0, T)^{N} .
\end{aligned}
$$

Now, by using (3.4) with $\varphi:=w_{j}-w$ and dividing by $\left\|v_{j}\right\|_{\mathbb{X}_{T}^{s}}$, we have

$$
\begin{aligned}
\left\langle w_{j}, w_{j}-w\right\rangle_{\mathbb{X}_{T}^{s}} & =\lambda_{\infty} \kappa_{s} \int_{\partial^{0} \mathcal{S}_{T}} \operatorname{Tr}\left(w_{j}\right)\left(\operatorname{Tr}\left(w_{j}\right)-\operatorname{Tr}(w)\right) d x \\
& -\kappa_{s} \int_{\partial^{0} \mathcal{S}_{T}} \frac{f\left(x, \operatorname{Tr}\left(v_{j}\right)\right)}{\left\|v_{j}\right\|_{\mathbb{X}_{T}^{s}}}\left(\operatorname{Tr}\left(w_{j}\right)-\operatorname{Tr}(w)\right) d x+o(1) .
\end{aligned}
$$

Moreover, by (3.8) we easily have

$\left|\int_{\partial^{0} \mathcal{S}_{T}} \operatorname{Tr}\left(w_{j}\right)\left(\operatorname{Tr}\left(w_{j}\right)-\operatorname{Tr}(w)\right) d x\right| \leqslant\left|\operatorname{Tr}\left(w_{j}\right)\right|_{2}\left|\operatorname{Tr}\left(w_{j}\right)-\operatorname{Tr}(w)\right|_{2}=o(1)$.

Now, relations (3.2), (3.7), and (3.8) yield 


$$
\begin{aligned}
\left|\int_{\partial^{0} \mathcal{S}_{T}} \frac{f\left(x, \operatorname{Tr}\left(v_{j}\right)\right)}{\left\|v_{j}\right\|_{\mathbb{X}_{T}^{s}}}\left(\operatorname{Tr}\left(w_{j}\right)-\operatorname{Tr}(w)\right) d x\right| \leqslant & \varepsilon \frac{\left|\operatorname{Tr}\left(w_{j}\right)\right|_{2}\left|\operatorname{Tr}\left(w_{j}\right)-\operatorname{Tr}(w)\right|_{2}}{\left\|v_{j}\right\|_{\mathbb{X}_{T}^{s}}} \\
& +\frac{a_{\varepsilon}\left|\operatorname{Tr}\left(w_{j}\right)-\operatorname{Tr}(w)\right|_{1}}{\left\|v_{j}\right\|_{\mathbb{X}_{T}^{s}}} \\
& =o(1)
\end{aligned}
$$

Putting together (3.9), (3.10) and (3.11) we get

$$
\left\langle w_{j}, w_{j}-w\right\rangle_{\mathbb{X}_{T}^{s}}=o(1) .
$$

Hence, in particular, we also have

$$
w_{j} \rightarrow w \quad \text { strongly in } \mathbb{X}_{T}^{s},
$$

and, by the definition of $w_{j}, w \neq 0$.

Now, dividing (3.6) by $\left\|v_{j}\right\|_{\mathbb{X}_{T}^{s}}$ we have

$$
\begin{aligned}
\left\langle w_{j}, \varphi\right\rangle_{\mathbb{X}_{T}^{s}}=\lambda_{\infty} \kappa_{s} & \int_{\partial^{0} \mathcal{S}_{T}} \operatorname{Tr}\left(w_{j}\right) \operatorname{Tr}(\varphi) d x \\
& -\kappa_{s} \int_{\partial^{0} \mathcal{S}_{T}} \frac{f\left(x, \operatorname{Tr}\left(v_{j}\right)\right)}{\left\|v_{j}\right\|_{\mathbb{X}_{T}^{s}}} \operatorname{Tr}(\varphi) d x+o(1) .
\end{aligned}
$$

Let us observe that (3.2), (3.7) and (3.8) give

$$
\lim _{j \rightarrow+\infty} \int_{\partial^{0} \mathcal{S}_{T}} \frac{f\left(x, \operatorname{Tr}\left(v_{j}\right)\right)}{\left\|v_{j}\right\|_{\mathbb{X}_{T}^{s}}} \operatorname{Tr}(\varphi) d x=0, \quad \forall \varphi \in \mathbb{X}_{T}^{s}
$$

Then, by using (3.12) and (3.14), and passing to the limit in (3.13) as $j \rightarrow+\infty$, we get

$$
\langle w, \varphi\rangle_{\mathbb{X}_{T}^{s}}=\lambda_{\infty} \kappa_{s} \int_{\partial^{0} \mathcal{S}_{T}} \operatorname{Tr}(w) \operatorname{Tr}(\varphi) d x, \quad \forall \varphi \in \mathbb{X}_{T}^{s}
$$

that is $u:=\operatorname{Tr}(w)$ is a weak solution of the following non-local problem

$$
\begin{cases}\left(-\Delta+m^{2}\right)^{s} u=\lambda u & \text { in }(0, T)^{N} \\ u\left(x+T e_{i}\right)=u(x) & \forall x \in \mathbb{R}^{N}, i \in \mathbb{Z}[1, N]\end{cases}
$$

which gives a contradiction because of $\lambda_{\infty} \notin \sigma\left(\left(-\Delta+m^{2}\right)^{s}\right)$. Thus we have proved that $\left\{\left\|v_{j}\right\|_{\mathbb{X}_{T}^{s}}\right\}_{j \in \mathbb{N}}$ is bounded.

Now it remains to check the validity of the Palais-Smale condition, that is we have to show that every Palais-Smale sequence for $\mathcal{J}$ strongly converges in $\mathbb{X}_{T}^{s}$, up to a subsequence.

Then, up to subsequence, there exists $v \in \mathbb{X}_{T}^{s}$ such that

$$
\begin{aligned}
& v_{j} \rightarrow v \quad \text { weakly in } \mathbb{X}_{T}^{s} \\
& \operatorname{Tr}\left(v_{j}\right) \rightarrow \operatorname{Tr}(v) \quad \text { strongly in } L^{2}(0, T)^{N} .
\end{aligned}
$$


By (3.5) and (3.15) one has

$$
\left\langle\mathcal{J}^{\prime}\left(v_{j}\right), v_{j}-v\right\rangle \rightarrow 0, \quad \text { as } j \rightarrow+\infty
$$

and, by (3.2), we also have

$$
\int_{\partial^{0} \mathcal{S}_{T}}\left|f\left(x, \operatorname{Tr}\left(v_{j}\right)\right)\right|\left|\operatorname{Tr}\left(v_{j}\right)-\operatorname{Tr}(v)\right| d x \rightarrow 0, \quad \text { as } j \rightarrow+\infty .
$$

Hence, by (3.4) and (3.16), it follows that

$$
\left\|v_{j}\right\|_{\mathbb{X}_{T}^{s}}^{2}-\left\langle v_{j}, v\right\rangle_{\mathbb{X}_{T}^{s}} \rightarrow 0
$$

as $j \rightarrow+\infty$. Then, by (3.15) and (3.18), one has

$$
v_{j} \rightarrow v \quad \text { strongly in } \mathbb{X}_{T}^{s} \text {. }
$$

The proof is now complete.

Taking into account that the (PS) compactness condition is satisfied, we can prove our first existence result.

Proof of Theorem 1. We aim to apply the Saddle Point Theorem due to Rabinowitz [32, Theorem 4.6]. Putting together (2.12), (3.3), and trace inequality, we have

$$
\mathcal{J}(v) \geqslant \frac{1}{2}\left(1-\frac{\lambda_{\infty}}{\lambda_{h+1}}-\frac{c_{1}}{\lambda_{h+1}}\right)\|v\|_{\mathbb{X}_{T}^{s}}^{2}-\kappa_{s} c_{1} T^{N},
$$

for every $v \in \mathbb{V}_{h}^{\perp}$.

Then we get

$$
\mathcal{J}(v) \geqslant c_{2}, \quad \forall v \in \mathbb{V}_{h}^{\perp}
$$

provided that $h$ large enough.

Now, by (3.2), we can see that fixed $\varepsilon>0$, we can find $C_{\varepsilon}>0$ such that

$$
\begin{aligned}
\mathcal{J}(v) \leqslant & \frac{1}{2}\|v\|_{\mathbb{X}_{T}^{s}}^{2}-\kappa_{s} \frac{\lambda_{\infty}}{2}|\operatorname{Tr}(v)|_{2}^{2} \\
& +\kappa_{s} \frac{\varepsilon}{2}|\operatorname{Tr}(v)|_{2}^{2}+\kappa_{s} C_{\varepsilon}|\operatorname{Tr}(v)|_{2},
\end{aligned}
$$

for every $v \in \mathbb{X}_{T}^{s}$.

Let $\lambda_{h}<\lambda_{\infty}$ and take $\varepsilon>0$ such that $\lambda_{h}+\varepsilon<\lambda_{\infty}$. Putting together (3.21) and (2.11), we obtain for any $v \in \mathbb{V}_{h}$

$$
\begin{aligned}
\mathcal{J}(v) \leqslant & \frac{\kappa_{s}}{2}\left(\lambda_{h}+\varepsilon-\lambda_{\infty}\right)|\operatorname{Tr}(v)|_{2}^{2} \\
& +\kappa_{s} C_{\varepsilon}|\operatorname{Tr}(v)|_{2} .
\end{aligned}
$$

By using (2.11), we can see that for $v \in \mathbb{V}_{h},|\operatorname{Tr}(v)|_{2} \rightarrow+\infty$ when $\|v\|_{\mathbb{X}_{T}^{s}} \rightarrow+\infty$, so (3.22) implies that $\mathcal{J}(v) \rightarrow-\infty$ as $\|v\|_{\mathbb{X}_{T}^{s}} \rightarrow+\infty$ and $v \in \mathbb{V}_{h}$. 
Therefore we can find a positive constant $c_{5}$ such that

$$
\mathcal{J}(v) \leqslant-c_{3}, \quad \forall v \in \mathbb{V}_{h} .
$$

Hence, the thesis follows by Proposition 1, (3.20) and (3.23).

Finally, if $\lambda_{\infty}<\lambda_{1}$, we can prove the existence of a weak solution for our problem by using direct minimization techniques.

\section{PROOF OF THEOREM 2}

This section is devoted to establish a multiplicity result for (1.2). Under our conditions on the nonlinear term $f$, one can obtain not only existence critical point theorems, but also sharper multiplicity results when the functionals are symmetric. In [10, 11, 12, 13], multiplicity results for critical points of even functionals are stated, and their proofs are based on the use of a pseudo-index theory; see, for instance, [9, 14] as general references on this topics.

Following this approach, we begin proving the following lemmas proving the necessary geometric features of the energy functional $\mathcal{J}$.

Lemma 2. Assume that conditions $\left(f_{1}\right)$ and $\left(f_{2}\right)$ hold. Let $\lambda_{h}$ be as in $\left(C_{\lambda_{\infty}}\right)$ and $\mathbb{V} \frac{\perp}{h-1}$ as defined in (2.9). Then, there exist two positive constants $\rho$ and $c_{0}$ such that, setting

$$
S_{\rho}:=\left\{v \in \mathbb{X}_{T}^{s}:\|v\|_{\mathbb{X}_{T}^{s}}=\rho\right\}
$$

the functional $\mathcal{J}$ in (3.1) verifies

$$
\mathcal{J}(v) \geqslant c_{0}, \quad \forall v \in S_{\rho} \cap \mathbb{V}_{h-1}^{\perp} .
$$

Proof. By using $\left(f_{2}\right)$ we know that

$$
\lim _{|t| \rightarrow+\infty} \frac{F(x, t)}{t^{2}}=0
$$

and

$$
\lim _{t \rightarrow 0} \frac{F(x, t)}{t^{2}}=\frac{\lambda_{0}}{2}
$$

uniformly with respect to almost every $x \in(0, T)^{N}$. Moreover, $\lambda_{0}<0$ in view of condition $\left(C_{\lambda_{\infty}}\right)$.

Then, for every $\varepsilon>0$ there exist $r_{\varepsilon}(\geqslant 1)$ and $\delta_{\varepsilon}>0$ such that

$$
|F(x, t)| \leqslant \frac{\varepsilon}{2} t^{2}, \quad \text { if }|t|>r_{\varepsilon}
$$

and

$$
\left|F(x, t)-\frac{\lambda_{0}}{2} t^{2}\right| \leqslant \frac{\varepsilon}{2} t^{2}, \quad \text { if }|t|<\delta_{\varepsilon}
$$

and for almost every $x \in(0, T)^{N}$. 
On the other hand, by $\left(f_{1}\right)$, taking any constant

$$
q \in\left[0, \frac{4 s}{N-2 s}\right)
$$

there exists $k_{r_{\varepsilon}}>0$ such that

$$
|F(x, t)| \leqslant k_{r_{\varepsilon}}|t|^{q+2}, \quad \text { if } \delta_{\varepsilon} \leqslant|t| \leqslant r_{\varepsilon},
$$

and for almost every $x \in(0, T)^{N}$.

Putting together (4.2)-(4.4), we can deduce that for any $\varepsilon>0$ there exists $k_{\varepsilon}>0$ such that

$$
F(x, t) \leqslant \frac{\lambda_{0}+\varepsilon}{2} t^{2}+k_{\varepsilon}|t|^{q+2},
$$

for almost every $x \in(0, T)^{N}$ and for all $t \in \mathbb{R}$.

As a consequence

$$
\int_{\partial^{0} \mathcal{S}_{T}} F(x, \operatorname{Tr}(v)) \mathrm{d} x \leqslant \frac{\lambda_{0}+\varepsilon}{2}|\operatorname{Tr}(v)|_{2}^{2}+k_{\varepsilon}|\operatorname{Tr}(v)|_{q+2}^{q+2},
$$

for every $v \in \mathbb{X}_{T}^{s}$.

From this and by using Theorem 4 , for a suitable $k_{\varepsilon}^{\prime}>0$ we can see that

$$
\mathcal{J}(v) \geqslant \frac{1}{2}\|v\|_{\mathbb{X}_{T}^{s}}^{2}-\frac{\kappa_{s}\left(\lambda_{\infty}+\lambda_{0}+\varepsilon\right)}{2}|\operatorname{Tr}(v)|_{2}^{2}-k_{\varepsilon}^{\prime}\|v\|_{\mathbb{X}_{T}^{s}}^{q+2},
$$

for every $v \in \mathbb{X}_{T}^{s}$.

Then, by (2.12) and (4.5), it follows that

$$
\mathcal{J}(v) \geqslant \frac{1}{2}\left(1-\frac{\lambda_{\infty}+\lambda_{0}+\varepsilon}{\lambda_{h}}\right)\|v\|_{\mathbb{X}_{T}^{s}}^{2}-k_{\varepsilon}^{\prime}\|v\|_{\mathbb{X}_{T}^{s}}^{q+2},
$$

for every $v \in \mathbb{V}_{h-1}^{\perp}$.

Hence, by $\left(C_{\lambda_{\infty}}\right)$, for a suitable $\varepsilon$, there exists $k_{\varepsilon}^{\prime \prime}>0$ such that

$$
\mathcal{J}(v) \geqslant k_{\varepsilon}^{\prime \prime}\|v\|_{\mathbb{X}_{T}^{s}}^{2}-k_{\varepsilon}^{\prime}\|v\|_{\mathbb{X}_{T}^{s}}^{q+2}
$$

for every $v \in \mathbb{V}_{h-1}^{\perp}$.

Thus we can find $\rho$ sufficiently small and $c_{0}>0$ such that inequality (4.1) holds.

Lemma 3. Assume that $\left(f_{1}\right)$ and (1.3) hold. Let $\lambda_{k}$ as in $\left(C_{\lambda_{\infty}}\right), \mathbb{V}_{k}$ as in (2.8) and $c_{0}$ as in Lemma 2. Then, there exists $c_{\infty}>c_{0}$ such that the functional $\mathcal{J}$ in (3.1) verifies

$$
\mathcal{J}(v) \leqslant c_{\infty}, \quad \forall v \in \mathbb{V}_{k}
$$


Proof. By (3.21), taking $\lambda_{k}$ as in $\left(C_{\lambda_{\infty}}\right)$ and $\varepsilon>0$ such that $\lambda_{k}+\varepsilon<\lambda_{\infty}$, it results that

$$
\mathcal{J}(v) \leqslant \frac{\kappa_{s}}{2}\left(\lambda_{k}+\varepsilon-\lambda_{\infty}\right)|\operatorname{Tr}(v)|_{2}^{2}+\kappa_{s} C_{\varepsilon}|\operatorname{Tr}(v)|_{2},
$$

for every $v \in \mathbb{V}_{k}$.

Then, in view of (2.11), there exists $c_{\infty}=c_{\infty}(\varepsilon)$ (with $c_{\infty}>c_{0}$ ), such that inequality (4.6) holds.

We conclude this section giving the proof of Theorem 2.

Proof of Theorem 2. The idea of the proof consists in applying [9, Theorem 2.9] (recalled in Subsection 2.3) to the functional $\mathcal{J}$ defined on the Hilbert space $\mathbb{X}_{T}^{s}$.

By Proposition 1, follows that $\mathcal{J}$ is an even functional satisfying (PS) in $\mathbb{R}$. Let us consider $\lambda_{h}, \mathbb{V} \frac{\perp}{h-1}, \rho, c_{0}$ as in Lemma 2 and $\lambda_{k}, \mathbb{V}_{k}, c_{\infty}$ as in Lemma 3.

Then we consider the pseudo-index theory $\left(S_{\rho} \cap \mathbb{V}_{h-1}^{\perp}, \mathcal{H}^{*}, \gamma^{*}\right)$ related to the genus, $S_{\rho} \cap \mathbb{V}_{h-1}^{\perp}$ and $\mathcal{J}$.

In view of Remark 2 , taking $V:=\mathbb{V}_{k}, \partial B:=S_{\rho}$ and $W:=\mathbb{V}_{h-1}^{\perp}$, we get

$$
\gamma\left(\mathbb{V}_{k} \cap h\left(S_{\rho} \cap \mathbb{V}_{h-1}^{\perp}\right)\right) \geqslant \operatorname{dim} \mathbb{V}_{k}-\operatorname{codim} \mathbb{V}_{h-1}^{\perp}, \quad \forall h \in \mathcal{H}^{*},
$$

which implies

$$
\gamma^{*}\left(\mathbb{V}_{k}\right) \geqslant k-h+1
$$

Then we can apply Theorem 6 with $\tilde{A}:=\mathbb{V}_{k}$ and $S:=S_{\rho} \cap \mathbb{V}_{h-1}^{\perp}$ to deduce that $\mathcal{J}$ has at least $k-h+1$ distinct pairs of critical points corresponding to at most $k-h+1$ distinct critical values $c_{i}$, where $c_{i}$ is as in (2.14).

Then, if $Z$ denotes the set of $k-h+1$ distinct pairs of critical points of $\mathcal{J}$ obtained applying Theorem 6 , the set $\operatorname{Tr}(Z)$ contains $k-h+1$ distinct pairs of weak solutions of problem (1.2). The proof is complete.

Remark 3. We point out that, combining the proof of our main results and those of [11, Theorem 3.1], Theorem 2 holds if we require that

$$
\lambda_{\infty}<\lambda_{h} \leqslant \lambda_{k}<\lambda_{0}+\lambda_{\infty}
$$

instead of condition $\left(C_{\lambda_{\infty}}\right)$.

As a matter of fact, we plan to consider further applications of our abstract framework for fractional equations involving a suitable resonant term in a forthcoming paper.

Acknowledgements. The paper has been carried out under the auspices of the INdAM - GNAMPA Project 2016 titled: Problemi variazionali su varietà Riemanniane e gruppi di Carnot. This manuscript 
was revised during the visit of G.M.B. to the Mathematics Section of the Abdus Salam International Centre for Theoretical Physics (ICTP) - Trieste, in August 2016. He would like to thank Prof. F. RodríguezVillegas for his kind invitation and warm hospitality during the visit at the ICTP. He also express his gratitude to Prof. S. Ouaro and the Research Group from Burkina Faso for the joint scientific activities in Trieste. A special thank goes to Ms. K. Mabilo and Prof. F. Maggi for their strong human support and generosity.

\section{REFERENCES}

[1] H. Amann and E. Zehnder, Nontrivial solutions for a class of nonresonance problems and applications to nonlinear differential equations, Ann. Scuola Norm. Sup. Pisa 7 (1980), 539-603.

[2] V. Ambrosio, Periodic solutions for a pseudo-relativistic Schrödinger equation, Nonlinear Anal. TMA 120 (2015), 262-284.

[3] V. Ambrosio, Periodic solutions for the non-local operator pseudo-relativistic $\left(-\Delta+m^{2}\right)^{s}-m^{2 s}$ with $m \geqslant 0$, DOI: 10.12775/TMNA.2016.063.

[4] V. Ambrosio, Periodic solutions for a superlinear fractional problem without the Ambrosetti-Rabinowitz condition, Discrete Contin. Dyn. Syst. 37 (2017), no. 5, 2265-2284.

[5] V. Ambrosio, Ground states solutions for a non-linear equation involving a pseudo-relativistic Schrödinger operator, J. Math. Phys. 57 (2016), no. 5, 051502, $18 \mathrm{pp}$.

[6] V. Ambrosio and G. Molica Bisci, Periodic solutions for nonlocal fractional equations, Commun. Pure Appl. Anal. 16 (2017), no. 1, 331-334.

[7] G. Autuori and P. Pucci, Existence of entire solutions for a class of quasilinear elliptic equations, NoDEA Nonlinear Differential Equations Appl. 20 (2013), 977-1009.

[8] G. Autuori and P. Pucci, Elliptic problems involving the fractional Laplacian in $\mathbb{R}^{N}$, J. Differential Equations 255 (2013), 2340-2362.

[9] P. Bartolo, V. Benci, and D. Fortunato, Abstract critical point theorems and applications to some nonlinear problems with "strong" resonance at infinity, Nonlinear Anal. 7 (1983), 981-1012.

[10] R. Bartolo, A.M. Candela, and A. Salvatore, p-Laplacian problems with nonlinearities interacting with the spectrum, Nonlinear Differ. Equ. Appl. 20 (2013), 1701-1721.

[11] R. Bartolo, A.M. Candela, and A. Salvatore, Perturbed asymptotically linear problems, Ann. Mat. Pura Appl. 193 (2014), 89-101.

[12] R. Bartolo and G. Molica Bisci, Asymptotically linear fractional p-Laplacian equations, Ann. Mat. Pura Appl. (in press).

[13] R. Bartolo and G. Molica Bisci, A pseudo-index approach to fractional equations, Expo. Math. 33 (2015), 502-516.

[14] V. Benci, On the critical point theory for indefinite functionals in the presence of symmetries, Trans. Am. Math. Soc. 274 (1982), 533-572.

[15] Z. Binlin, G. Molica Bisci, and R. Servadei, Superlinear nonlocal fractional problems with infinitely many solutions, Nonlinearity 28 (2015), 2247-2264. 
[16] C. Brändle, E. Colorado, A. de Pablo, and U. Sánchez, A concave-convex elliptic problem involving the fractional Laplacian, Proc. Roy. Soc. Edinburgh Sect. A 143 (2013), 39-71.

[17] X. Cabré and J. Tan, Positive solutions of nonlinear problems involving the square root of the Laplacian, Adv. Math. 224 (2010), 2052-2093.

[18] L.A. Caffarelli and L. Silvestre, An extension problem related to the fractional Laplacian, Comm. Partial Differential Equations 32 (2007), 1245-1260.

[19] R. Carmona, W.C. Masters, and B. Simon, Relativistic Schrödinger operators: Asymptotic behavior of the eigenfunctions, J. Func. Anal 91 (1990), 117-142.

[20] E. Di Nezza, G. Palatucci, and E. Valdinoci, Hitchhiker's guide to the fractional Sobolev spaces, Bull. Sci. Math. 136 (2012), 521-573.

[21] A. Fiscella, Saddle point solutions for non-local elliptic operators, preprint, arXiv: 1210.8401 .

[22] A. Fiscella, R. Servadei, and E. Valdinoci, A resonance problem for non-local elliptic operators, Z. Anal. Anwendungen 32 (2013), 411-431.

[23] T. Kuusi, G. Mingione, and Y. Sire, Nonlocal equations with measure data, Comm. Math. Phys. 337 (2015), 1317-1368.

[24] T. Kuusi, G. Mingione, and Y. Sire, Nonlocal self-improving properties, Anal. PDE 8 (2015) 57-114.

[25] E.H. Lieb and M. Loss, Analysis, American Mathematical Society, Providence, RI, 2001.

[26] G. Molica Bisci, V. Rădulescu, and R. Servadei, Variational Methods for Nonlocal Fractional Problems. With a Foreword by Jean Mawhin, Encyclopedia of Mathematics and its Applications, Cambridge University Press 162, Cambridge, 2016. ISBN 9781107111943.

[27] G. Molica Bisci, V. Rădulescu, and R. Servadei, Nontrivial solutions of superlinear nonlocal problems, Forum Math. (in press).

[28] G. Palatucci and A. Pisante, Improved Sobolev embeddings, profile decomposition, and concentration-compactness for fractional Sobolev spaces, Calc. Var. Partial Differential Equations 50 (2014), 799-829.

[29] G. Palatucci and A. Pisante, A global compactness type result for Palais-Smale sequences in fractional Sobolev spaces, Nonlinear Anal. 117 (2015), 1-7.

[30] P. Pucci and S. Saldi, Multiple solutions for an eigenvalue problem involving non-local elliptic p-Laplacian operators, in Geometric Methods in PDE's Springer INdAM Series - Vol. 11, G. Citti, M. Manfredini, D. Morbidelli, S. Polidoro, F. Uguzzoni Eds., pages 16.

[31] P. Pucci and S. Saldi, Critical stationary Kirchhoff equations in $\mathbb{R}^{N}$ involving nonlocal operators, to appear in Rev. Mat. Iberoam., pages 23.

[32] P.H. Rabinowitz, Minimax Methods in Critical Point Theory with Applications to Differential Equations, CBMS Regional Conf. Ser. in Math. 65, Amer. Math. Soc., Providence, 1984.

[33] F. Riesz and B. Szökefalvi-Nagy, Lecons d'analyse fonctionnelle, Académie des Sciences de Hongrie, Akadémiai Kiadó, Budapest, (1952). viii+449 pp.

[34] M. Ryznar, Estimate of Green function for relativistic $\alpha$-stable processes, Potential Analysis, 17, (2002), 1-23.

[35] S. Secchi, Ground state solutions for nonlinear fractional Schrödinger equations in $\mathbb{R}^{N}$, J. Math. Phys. 54, 031501 (2013). 
[36] S. Secchi, Perturbation results for some nonlinear equations involving fractional operators, Differ. Equ. Appl. 5 (2013), 221-236.

[37] R. Servadei and E. Valdinoci, Mountain Pass solutions for non-local elliptic operators, J. Math. Anal. Appl. 389 (2012), 887-898.

[38] R. Servadei and E. Valdinoci, Variational methods for non-local operators of elliptic type, Discrete Contin. Dyn. Syst. 33 (2013), 2105-2137.

[39] M. Struwe, Variational Methods. Applications to Nonlinear Partial Differential Equations and Hamiltonian Systems, 4rd Edition, Ergeb. Math. Grenzgeb. (4) 34, Springer-Verlag, Berlin, 2008.

[40] J.L. Vázquez, Nonlinear Diffusion with Fractional Laplacian Operators, in Nonlinear Partial Differential Equations, Abel Symp. 7 (2012), 271-298.

[41] K. Yosida Functional Analysis, Die Grundlehren der Mathematischen Wissenschaften, Band 123 Academic Press, Inc., New York; Springer-Verlag, Berlin (1965) xi+458 pp.

VinCENZO AMBRosio

Dipartimento di Scienze Pure e Applicate (DiSPeA)

Università degli Studi di URbino 'CARLo Bo'

Piazza della Repubblica, 13

61029 Urbino (Pesaro e Urbino)

ITALY

E-mail address: vincenzo.ambrosio@uniurb.it

Giovanni Molica Bisci

DipARTIMENTO PAU

Università 'Mediterranea' di Reggio Calabria

Salita Melissari, Feo di Vito, 89100 Reggio Calabria

ITALY

E-mail address: gmolica@unirc.it 